\title{
Some Properties of Membership Functions Composed of Triangle Functions and Piecewise Linear Functions
}

\author{
Takashi Mitsuishi \\ University of Marketing and Distribution Sciences \\ Kobe, Japan
}

\begin{abstract}
Summary. IF-THEN rules in fuzzy inference is composed of multiple fuzzy sets (membership functions). IF-THEN rules can therefore be considered as a pair of membership functions [7. The evaluation function of fuzzy control is composite function with fuzzy approximate reasoning and is functional on the set of membership functions. We obtained continuity of the evaluation function and compactness of the set of membership functions [12. Therefore, we proved the existence of pair of membership functions, which maximizes (minimizes) evaluation function and is considered IF-THEN rules, in the set of membership functions by using extreme value theorem. The set of membership functions (fuzzy sets) is defined in this article to verifier our proofs before by Mizar [9], 10], 4]. Membership functions composed of triangle function, piecewise linear function and Gaussian function used in practice are formalized using existing functions.

On the other hand, not only curve membership functions mentioned above but also membership functions composed of straight lines (piecewise linear function) like triangular and trapezoidal functions are formalized. Moreover, different from the definition in [3] formalizations of triangular and trapezoidal function composed of two straight lines, minimum function and maximum functions are proposed. We prove, using the Mizar 2, 1] formalism, some properties of membership functions such as continuity and periodicity [13, 8].
\end{abstract}

MSC: 03E72 68V20

Keywords: membership function; piecewise linear function

MML identifier: FUZZY_5, version: 8.1.11 5.66.1402

\footnotetext{
${ }^{1}$ This work has been partially supported in $2019-2020$ by the domestic research grant of University of Marketing and Distribution Sciences in Kobe (Japan). 


\section{Preliminaries}

Now we state the propositions:

(1) Let us consider real numbers $a, b, c, d$. Then $\mid \max (c, \min (d, a))-\max (c$, $\min (d, b))|\leqslant| a-b \mid$.

(2) Let us consider a real number $x$. Then $|\sin x| \leqslant|x|$.

(3) Let us consider real numbers $x, y$. Then $|\sin x-\sin y| \leqslant|x-y|$. The theorem is a consequence of (2).

(4) Let us consider a real number $x$. If $\exp x=1$, then $x=0$.

(5) Let us consider real numbers $a, b, p, q$. Suppose $a>0$ and $p>0$ and $\frac{-b}{a}<\frac{q}{p}$. Then

(i) $\frac{-b}{a}<\frac{q-b}{a+p}<\frac{q}{p}$, and

(ii) $\frac{a \cdot q+b \cdot p}{a+p}>0$.

(6) Let us consider real numbers $a, b, p, q, s$. Suppose $a>0$ and $p>0$ and $\frac{s-b}{a}=\frac{s-q}{-p}$. Then

(i) $\frac{s-b}{a}=\frac{q-b}{a+p}$, and

(ii) $\frac{s-q}{-p}=\frac{q-b}{a+p}$.

(7) Let us consider real numbers $a, b, p, q, s$. Suppose $a>0$ and $p>0$ and $\frac{s-b}{a}<\frac{s-q}{-p}$. Then $\frac{s-b}{a}<\frac{q-b}{a+p}<\frac{s-q}{-p}$.

\section{The Set of Membership Functions}

Let $X$ be a non empty set. The functor Membership-Funcs $(X)$ yielding a set is defined by

(Def. 1) for every object $f, f \in i t$ iff $f$ is a membership function of $X$.

Now we state the propositions:

(8) Let us consider a non empty set $X$, and an object $x$. Suppose $x \in$ Membership-Funcs $(X)$. Then there exists a membership function $f$ of $X$ such that

(i) $x=f$, and

(ii) $\operatorname{dom} f=X$.

(9) Membership-Funcs $(\mathbb{R})=\{f$, where $f$ is a function from $\mathbb{R}$ into $\mathbb{R}: f$ is a fuzzy set of $\mathbb{R}\}$. The theorem is a consequence of (8).

(10) Let us consider non empty sets $A, X$.

Then $\left\{\chi_{A, X}\right\} \subseteq$ Membership-Funcs $(X)$. 
(11) $\left\{\chi_{[a, b], \mathbb{R}}\right.$, where $a, b$ are real numbers : $\left.a \leqslant b\right\} \subseteq \operatorname{Membership-Funcs}(\mathbb{R})$.

(12) $\left\{\chi_{A, \mathbb{R}}\right.$, where $A$ is a subset of $\left.\mathbb{R}: A \subseteq \mathbb{R}\right\} \subseteq \operatorname{Membership-Funcs}(\mathbb{R})$.

(13) $\{f$, where $f$ is a fuzzy set of $\mathbb{R}$ : there exists a subset $A$ of $\mathbb{R}$ such that $\left.f=\chi_{A, \mathbb{R}}\right\} \subseteq \operatorname{Membership-Funcs}(\mathbb{R})$.

(14) Let us consider functions $f, g$ from $\mathbb{R}$ into $\mathbb{R}$, and a real number $a$. Suppose $g$ is continuous and for every real number $x, f(x)=\min (a, g(x))$. Then $f$ is continuous.

Proof: For every real number $x_{0}$ such that $x_{0} \in \operatorname{dom} f$ holds $f$ is continuous in $x_{0}$.

Let us consider functions $F, f, g$ from $\mathbb{R}$ into $\mathbb{R}$. Now we state the propositions:

(15) If $f$ is continuous and $g$ is continuous and for every real number $x$, $F(x)=\min (f(x), g(x))$, then $F$ is continuous.

Proof: For every real number $x_{0}$ such that $x_{0} \in \operatorname{dom} F$ holds $F$ is continuous in $x_{0}$.

(16) If $f$ is continuous and $g$ is continuous and for every real number $x$, $F(x)=\max (f(x), g(x))$, then $F$ is continuous.

Proof: For every real number $x_{0}$ such that $x_{0} \in \operatorname{dom} F$ holds $F$ is continuous in $x_{0}$.

(17) Let us consider functions $f, g$ from $\mathbb{R}$ into $\mathbb{R}$, and a real number $a$. Suppose $g$ is continuous and for every real number $x, f(x)=\max (a, g(x))$. Then $f$ is continuous. The theorem is a consequence of (16).

(18) Let us consider functions $f, g$ from $\mathbb{R}$ into $\mathbb{R}$, and real numbers $a, b$. Suppose $g$ is continuous and for every real number $x, f(x)=\max (a, \min (b$, $g(x)))$. Then $f$ is continuous.

Proof: Define $\mathcal{H}($ element of $\mathbb{R})=\left(\min \left(b, g\left(\$_{1}\right)\right)\right)(\in \mathbb{R})$. Consider $h$ being a function from $\mathbb{R}$ into $\mathbb{R}$ such that for every element $x$ of $\mathbb{R}, h(x)=\mathcal{H}(x)$. For every real number $x, h(x)=\min (b, g(x))$. $h$ is continuous. For every real number $x, f(x)=\max (a, h(x))$.

(19) Let us consider functions $f, g$ from $\mathbb{R}$ into $\mathbb{R}$. Suppose $g$ is continuous and for every real number $x, f(x)=\max (0, \min (1, g(x)))$. Then $f$ is continuous.

Let us consider a function $f$ from $\mathbb{R}$ into $\mathbb{R}$ and real numbers $a, b$. Now we state the propositions:

(20) If for every real number $t_{1}, f\left(t_{1}\right)=\frac{1}{2} \cdot\left(\sin \left(a \cdot t_{1}+b\right)\right)+\frac{1}{2}$, then $f$ is continuous.

Proof: For every real number $x_{0}$ such that $x_{0} \in \operatorname{dom} f$ holds $f$ is continuous in $x_{0}$. 
(21) If for every real number $x, f(x)=\frac{1}{2} \cdot(\sin (a \cdot x+b))+\frac{1}{2}$, then $f$ is continuous.

(22) Let us consider real numbers $r, s$, and a function $f$ from $\mathbb{R}$ into $\mathbb{R}$. Suppose for every real number $x, f(x)=\max (r, \min (s, x))$. Then $f$ is Lipschitzian. The theorem is a consequence of (1).

(23) Let us consider a function $g$ from $\mathbb{R}$ into $\mathbb{R}$. Then $\{f$, where $f$ is a function from $\mathbb{R}$ into $\mathbb{R}$ : for every real number $x, f(x)=\min (1, \max (0, g(x)))\} \subseteq$ $\operatorname{Membership-Funcs(}(\mathbb{R})$.

Proof: Consider $f$ being a function from $\mathbb{R}$ into $\mathbb{R}$ such that $f_{0}=f$ and for every real number $x, f(x)=\min (1, \max (0, g(x)))$. $\operatorname{rng} f \subseteq[0,1]$.

(24) $\{f$, where $f, g$ are functions from $\mathbb{R}$ into $\mathbb{R}$ : for every real number $x, f(x)$ $=\max (0, \min (1, g(x)))\} \subseteq$ Membership-Funcs $(\mathbb{R})$.

Let us consider functions $f, g$ from $\mathbb{R}$ into $\mathbb{R}$. Now we state the propositions:

(25) If for every real number $x, f(x)=\max (0, \min (1, g(x)))$, then $f$ is a fuzzy set of $\mathbb{R}$.

(26) If for every real number $x, f(x)=\min (1, \max (0, g(x)))$, then $f$ is a fuzzy set of $\mathbb{R}$. The theorem is a consequence of $(23)$.

(27) $\{f$, where $f$ is a function from $\mathbb{R}$ into $\mathbb{R}$ : there exist real numbers $a, b$ such that for every real number $\left.t_{1}, f\left(t_{1}\right)=\frac{1}{2} \cdot\left(\sin \left(a \cdot t_{1}+b\right)\right)+\frac{1}{2}\right\} \subseteq$ Membership-Funcs(R).

Proof: Consider $f$ being a function from $\mathbb{R}$ into $\mathbb{R}$ such that $x=f$ and there exist real numbers $a, b$ such that for every real number $t_{1}$, $f\left(t_{1}\right)=\frac{1}{2} \cdot\left(\sin \left(a \cdot t_{1}+b\right)\right)+\frac{1}{2} \cdot \operatorname{rng} f \subseteq[0,1]$.

(28) $\{f$, where $f$ is a function from $\mathbb{R}$ into $\mathbb{R}, a, b$ are real numbers : for every real number $\left.t_{1}, f\left(t_{1}\right)=\frac{1}{2} \cdot\left(\sin \left(a \cdot t_{1}+b\right)\right)+\frac{1}{2}\right\} \subseteq \operatorname{Membership-Funcs~}(\mathbb{R})$.

Proof: Consider $f$ being a function from $\mathbb{R}$ into $\mathbb{R}, a, b$ being real numbers such that $x=f$ and for every real number $t_{1}, f\left(t_{1}\right)=\frac{1}{2} \cdot\left(\sin \left(a \cdot t_{1}+b\right)\right)+\frac{1}{2}$. $\operatorname{rng} f \subseteq[0,1]$.

(29) Let us consider real numbers $a, b$, and a function $f$ from $\mathbb{R}$ into $\mathbb{R}$. Suppose for every real number $t_{1}, f\left(t_{1}\right)=\frac{1}{2} \cdot\left(\sin \left(a \cdot t_{1}+b\right)\right)+\frac{1}{2}$. Then $f$ is a fuzzy set of $\mathbb{R}$. The theorem is a consequence of (28).

(30) $\{f$, where $f$ is a function from $\mathbb{R}$ into $\mathbb{R}$ : there exist real numbers $a, b$ such that for every real number $\left.t_{1}, f\left(t_{1}\right)=\frac{1}{2} \cdot\left(\cos \left(a \cdot t_{1}+b\right)\right)+\frac{1}{2}\right\} \subseteq$ Membership-Funcs(R).

Proof: Consider $f$ being a function from $\mathbb{R}$ into $\mathbb{R}$ such that $x=f$ and there exist real numbers $a, b$ such that for every real number $t_{1}$, $f\left(t_{1}\right)=\frac{1}{2} \cdot\left(\cos \left(a \cdot t_{1}+b\right)\right)+\frac{1}{2} \cdot \operatorname{rng} f \subseteq[0,1]$.

(31) Let us consider real numbers $a, b$, and a function $f$ from $\mathbb{R}$ into $\mathbb{R}$. 
Suppose for every real number $t_{1}, f\left(t_{1}\right)=\frac{1}{2} \cdot\left(\cos \left(a \cdot t_{1}+b\right)\right)+\frac{1}{2}$. Then $f$ is a fuzzy set of $\mathbb{R}$. The theorem is a consequence of $(30)$.

(32) Let us consider real numbers $a, b$, and a fuzzy set $f$ of $\mathbb{R}$. Suppose $a \neq 0$ and for every real number $t_{1}, f\left(t_{1}\right)=\frac{1}{2} \cdot\left(\sin \left(a \cdot t_{1}+b\right)\right)+\frac{1}{2}$. Then $f$ is normalized.

Proof: There exists an element $x$ of $\mathbb{R}$ such that $f(x)=1$.

(33) Let us consider a fuzzy set $f$ of $\mathbb{R}$. Suppose $f \in\{f$, where $f$ is a function from $\mathbb{R}$ into $\mathbb{R}$ : there exist real numbers $a, b$ such that $a \neq 0$ and for every real number $\left.t_{1}, f\left(t_{1}\right)=\frac{1}{2} \cdot\left(\sin \left(a \cdot t_{1}+b\right)\right)+\frac{1}{2}\right\}$. Then $f$ is normalized.

ProOF: Consider $f_{2}$ being a function from $\mathbb{R}$ into $\mathbb{R}$ such that $f=f_{2}$ and there exist real numbers $a, b$ such that $a \neq 0$ and for every real number $t_{1}, f_{2}\left(t_{1}\right)=\frac{1}{2} \cdot\left(\sin \left(a \cdot t_{1}+b\right)\right)+\frac{1}{2}$. Consider $a, b$ being real numbers such that $a \neq 0$ and for every real number $t_{1}, f_{2}\left(t_{1}\right)=\frac{1}{2} \cdot\left(\sin \left(a \cdot t_{1}+b\right)\right)+\frac{1}{2}$. There exists an element $x$ of $\mathbb{R}$ such that $f(x)=1$.

(34) Let us consider a fuzzy set $f$ of $\mathbb{R}$, and real numbers $a, b$. Suppose $a \neq 0$ and for every real number $t_{1}, f\left(t_{1}\right)=\frac{1}{2} \cdot\left(\cos \left(a \cdot t_{1}+b\right)\right)+\frac{1}{2}$. Then $f$ is normalized.

Proof: There exists an element $x$ of $\mathbb{R}$ such that $f(x)=1$.

(35) Let us consider a fuzzy set $f$ of $\mathbb{R}$. Suppose $f \in\{f$, where $f$ is a function from $\mathbb{R}$ into $\mathbb{R}$ : there exist real numbers $a, b$ such that $a \neq 0$ and for every real number $\left.t_{1}, f\left(t_{1}\right)=\frac{1}{2} \cdot\left(\cos \left(a \cdot t_{1}+b\right)\right)+\frac{1}{2}\right\}$. Then $f$ is normalized. The theorem is a consequence of (34).

(36) Let us consider a function $F$ from $\mathbb{R}$ into $\mathbb{R}$, real numbers $a, b, c, d$, and an integer $i$. Suppose $a \neq 0$ and $i \neq 0$ and for every real number $x$, $F(x)=\max (0, \min (1, c \cdot(\sin (a \cdot x+b))+d))$. Then $F$ is $\left(\frac{2 \cdot \pi}{a} \cdot i\right)$-periodic. Proof: For every real number $x$ such that $x \in \operatorname{dom} F \operatorname{holds} x+\frac{2 \cdot \pi}{a} \cdot i$, $x-\frac{2 \cdot \pi}{a} \cdot i \in \operatorname{dom} F$ and $F(x)=F\left(x+\frac{2 \cdot \pi}{a} \cdot i\right)$.

(37) Let us consider a function $F$ from $\mathbb{R}$ into $\mathbb{R}$, and real numbers $a, b, c$, d. Suppose for every real number $x, F(x)=\max (0, \min (1, c \cdot(\sin (a \cdot x+$ $b))+d)$ ). Then $F$ is periodic.

Proof: There exists a real number $t$ such that $F$ is $t$-periodic by $(36), 6$, (1)].

(38) $\{f$, where $f$ is a function from $\mathbb{R}$ into $\mathbb{R}$ : there exist real numbers $a, b$ such that for every real number $\left.t_{1}, f\left(t_{1}\right)=\max \left(0, \sin \left(a \cdot t_{1}+b\right)\right)\right\} \subseteq$ $\operatorname{Membership-Funcs}(\mathbb{R})$.

Proof: Consider $f$ being a function from $\mathbb{R}$ into $\mathbb{R}$ such that $x=f$ and there exist real numbers $a, b$ such that for every real number $t_{1}$, $f\left(t_{1}\right)=\max \left(0, \sin \left(a \cdot t_{1}+b\right)\right) \cdot \operatorname{rng} f \subseteq[0,1]$ by $[\underline{5},(4)]$.

(39) Let us consider real numbers $a, b$, and a function $f$ from $\mathbb{R}$ into $\mathbb{R}$. 
Suppose for every real number $x, f(x)=\max (0, \sin (a \cdot x+b))$. Then $f$ is a fuzzy set of $\mathbb{R}$. The theorem is a consequence of (38).

(40) $\{f$, where $f$ is a function from $\mathbb{R}$ into $\mathbb{R}$ : there exist real numbers $a, b$ such that for every real number $x, f(x)=\max (0, \cos (a \cdot x+b))\} \subseteq$ Membership-Funcs $(\mathbb{R})$.

Proof: Consider $f$ being a function from $\mathbb{R}$ into $\mathbb{R}$ such that $x=f$ and there exist real numbers $a, b$ such that for every real number $t_{1}$, $f\left(t_{1}\right)=\max \left(0, \cos \left(a \cdot t_{1}+b\right)\right) \cdot \operatorname{rng} f \subseteq[0,1]$.

(41) Let us consider real numbers $a, b$, and a function $f$ from $\mathbb{R}$ into $\mathbb{R}$. Suppose for every real number $x, f(x)=\max (0, \cos (a \cdot x+b))$. Then $f$ is a fuzzy set of $\mathbb{R}$. The theorem is a consequence of (40).

(42) $\{f$, where $f$ is a function from $\mathbb{R}$ into $\mathbb{R}, a, b, c, d$ are real numbers : for every real number $x, f(x)=\max (0, \min (1, c \cdot(\sin (a \cdot x+b))+d))\} \subseteq$ $\{f$, where $f, g$ are functions from $\mathbb{R}$ into $\mathbb{R}$ : for every real number $x, f(x)=$ $\max (0, \min (1, g(x)))\}$.

(43) $\{f$, where $f$ is a function from $\mathbb{R}$ into $\mathbb{R}, a, b, c, d$ are real numbers : for every real number $x, f(x)=\max (0, \min (1, c \cdot(\sin (a \cdot x+b))+d))\} \subseteq$

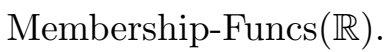

Proof: Consider $f$ being a function from $\mathbb{R}$ into $\mathbb{R}, a, b, c, d$ being real numbers such that $f=g$ and for every real number $x, f(x)=$ $\max (0, \min (1, c \cdot(\sin (a \cdot x+b))+d)) . f$ is a fuzzy set of $\mathbb{R}$.

(44) Let us consider a function $f$ from $\mathbb{R}$ into $\mathbb{R}$, and real numbers $a, b, c, d$. Suppose for every real number $x, f(x)=\max (0, \min (1, c \cdot(\sin (a \cdot x+b))+$ $d)$ ). Then $f$ is a fuzzy set of $\mathbb{R}$. The theorem is a consequence of (43).

(45) $\{f$, where $f$ is a function from $\mathbb{R}$ into $\mathbb{R}, a, b, c, d$ are real numbers : for every real number $x, f(x)=\max (0, \min (1, c \cdot(\arctan (a \cdot x+b))+d))\} \subseteq$ $\{f$, where $f, g$ are functions from $\mathbb{R}$ into $\mathbb{R}$ : for every real number $x, f(x)=$ $\max (0, \min (1, g(x)))\}$.

(46) $\{f$, where $f$ is a function from $\mathbb{R}$ into $\mathbb{R}, a, b, c, d$ are real numbers : for every real number $x, f(x)=\max (0, \min (1, c \cdot(\arctan (a \cdot x+b))+d))\} \subseteq$

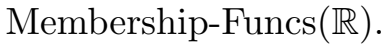

(47) Let us consider a function $f$ from $\mathbb{R}$ into $\mathbb{R}$, and real numbers $a, b, c, d$. Suppose for every real number $x, f(x)=\max (0, \min (1, c \cdot(\arctan (a \cdot x+$ $b))+d)$ ). Then $f$ is a fuzzy set of $\mathbb{R}$. The theorem is a consequence of (68) and (24).

(48) Let us consider a function $f$ from $\mathbb{R}$ into $\mathbb{R}$, and real numbers $a, b, c, d$, $r, s$. Suppose for every real number $x, f(x)=\max (r, \min (s, c \cdot(\sin (a \cdot x+$ $b))+d)$ ). Then $f$ is Lipschitzian.

Proof: There exists a real number $r$ such that $0<r$ and for every real 
numbers $x_{1}, x_{2}$ such that $x_{1}, x_{2} \in \operatorname{dom} f$ holds $\left|f\left(x_{1}\right)-f\left(x_{2}\right)\right| \leqslant r \cdot\left|x_{1}-x_{2}\right|$.

(49) Let us consider a function $f$ from $\mathbb{R}$ into $\mathbb{R}$, and real numbers $a, b, c, d$. Suppose for every real number $x, f(x)=\max (0, \min (1, c \cdot(\sin (a \cdot x+b))+$ d)). Then $f$ is Lipschitzian.

Let us consider real numbers $a, b$ and a function $f$ from $\mathbb{R}$ into $\mathbb{R}$. Now we state the propositions:

(50) If $b \neq 0$ and for every real number $x, f(x)=\exp \left(-\frac{(x-a)^{2}}{2 \cdot b^{2}}\right)$, then $f$ is a fuzzy set of $\mathbb{R}$.

Proof: $\operatorname{rng} f \subseteq[0,1]$.

(51) If $b \neq 0$ and for every real number $x, f(x)=\exp \left(-\frac{(x-a)^{2}}{2 \cdot b^{2}}\right)$, then $f$ is a fuzzy set of $\mathbb{R}$.

PROOF: For every real number $x, f(x)=\exp \left(-\frac{(x-a)^{2}}{2 \cdot b^{2}}\right)$.

(52) Let us consider real numbers $a, b$. Suppose $b \neq 0$. Then $\{f$, where $f$ is a function from $\mathbb{R}$ into $\mathbb{R}$ : for every real number $\left.x, f(x)=\exp \left(-\frac{(x-a)^{2}}{2 \cdot b^{2}}\right)\right\}$ $\subseteq$ Membership-Funcs $(\mathbb{R})$. The theorem is a consequence of (51).

Let us consider real numbers $a, b$ and a fuzzy set $f$ of $\mathbb{R}$. Now we state the propositions:

(53) If for every real number $x, f(x)=\exp \left(-\frac{(x-a)^{2}}{2 \cdot b^{2}}\right)$, then $f$ is normalized.

Proof: There exists an element $x$ of $\mathbb{R}$ such that $f(x)=1$.

(54) If $b \neq 0$ and for every real number $x, f(x)=\exp \left(-\frac{(x-a)^{2}}{2 \cdot b^{2}}\right)$, then $f$ is strictly normalized.

Proof: There exists an element $x$ of $\mathbb{R}$ such that $f(x)=1$ and for every element $y$ of $\mathbb{R}$ such that $f(y)=1$ holds $y=x$ by [11, (20)], (4).

(55) Let us consider real numbers $a, b$, and a function $f$ from $\mathbb{R}$ into $\mathbb{R}$. Suppose $b \neq 0$ and for every real number $x, f(x)=\exp \left(-\frac{(x-a)^{2}}{2 \cdot b^{2}}\right)$. Then $f$ is continuous.

Proof: Set $h=\operatorname{AffineMap}(1,-a) \cdot f=($ the function $\exp ) \cdot\left(\left(\frac{-1}{2 \cdot b^{2}} \cdot h\right) \cdot h\right)$.

(56) Let us consider real numbers $a, b, c, r, s$, and a function $f$ from $\mathbb{R}$ into $\mathbb{R}$. Suppose $b \neq 0$ and for every real number $x, f(x)=$ $\max \left(r, \min \left(s, \exp \left(-\frac{(x-a)^{2}}{2 \cdot b^{2}}\right)+c\right)\right)$. Then $f$ is continuous.

Proof: Define $\mathcal{H}($ element of $\mathbb{R})=\left(\exp \left(-\frac{\left(\$_{1}-a\right)^{2}}{2 \cdot b^{2}}\right)\right)(\in \mathbb{R})$. Consider $h$ being a function from $\mathbb{R}$ into $\mathbb{R}$ such that for every element $x$ of $\mathbb{R}, h(x)=\mathcal{H}(x)$. For every real number $x_{0}$ such that $x_{0} \in \operatorname{dom} f$ holds $f$ is continuous in $x_{0}$. 
Let us consider real numbers $a, b, c$ and a function $f$ from $\mathbb{R}$ into $\mathbb{R}$. Now we state the propositions:

(57) Suppose $b \neq 0$ and for every real number $x, f(x)=$ $\max \left(0, \min \left(1, \exp \left(-\frac{(x-a)^{2}}{2 \cdot b^{2}}\right)+c\right)\right)$. Then $f$ is continuous.

(58) Suppose $b \neq 0$ and for every real number $x, f(x)=$ $\max \left(0, \min \left(1, \exp \left(-\frac{(x-a)^{2}}{2 \cdot b^{2}}\right)+c\right)\right)$. Then $f$ is a fuzzy set of $\mathbb{R}$. The theorem is a consequence of $(25)$.

(59) $\{f$, where $f$ is a function from $\mathbb{R}$ into $\mathbb{R}, a, b, c$ are real numbers : $b \neq$ 0 and for every real number $\left.x, f(x)=\max \left(0, \min \left(1, \exp \left(-\frac{(x-a)^{2}}{2 \cdot b^{2}}\right)+c\right)\right)\right\}$ $\subseteq$ Membership-Funcs $(\mathbb{R})$. The theorem is a consequence of $(58)$.

(60) Let us consider a function $f$ from $\mathbb{R}$ into $\mathbb{R}$, and real numbers $a, b, r, s$. Suppose for every real number $x, f(x)=$ $\max (r, \min (s,(\operatorname{AffineMap}(a, b))(x)))$. Then $f$ is Lipschitzian.

Proof: There exists a real number $r$ such that $0<r$ and for every real numbers $x_{1}, x_{2}$ such that $x_{1}, x_{2} \in \operatorname{dom} f$ holds $\left|f\left(x_{1}\right)-f\left(x_{2}\right)\right| \leqslant r \cdot\left|x_{1}-x_{2}\right|$.

Let us consider a function $f$ from $\mathbb{R}$ into $\mathbb{R}$ and real numbers $a, b$. Now we state the propositions:

(61) If for every real number $x, f(x)=\max (0, \min (1,(\operatorname{AffineMap}(a, b))(x)))$, then $f$ is Lipschitzian.

(62) If for every real number $x, f(x)=\max (0, \min (1,(\operatorname{AffineMap}(a, b))(x)))$, then $f$ is a fuzzy set of $\mathbb{R}$.

(63) $\{f$, where $f$ is a function from $\mathbb{R}$ into $\mathbb{R}, a, b$ are real numbers : for every real number $x, f(x)=\max (0, \min (1,(\operatorname{AffineMap}(a, b))(x)))\} \subseteq$ Membership-Funcs $(\mathbb{R})$. The theorem is a consequence of $(25)$.

(64) Let us consider real numbers $a, b$, and a function $f$ from $\mathbb{R}$ into $\mathbb{R}$. Suppose for every real number $x, f(x)=\max \left(0,1-\left|\frac{x-a}{b}\right|\right)$. Then $f$ is a fuzzy set of $\mathbb{R}$.

Proof: $\operatorname{rng} f \subseteq[0,1]$.

(65) Let us consider real numbers $a, b$. Suppose $b>0$. Let us consider a real number $x$. Then $(\operatorname{TriangularFS}((a-b), a,(a+b)))(x)=\max \left(0,1-\left|\frac{x-a}{b}\right|\right)$. Proof: Set $\left.f_{1}=(\operatorname{AffineMap}(0,0)) \mid \mathbb{R} \backslash\right] a-b, a+b[$.

Set $f_{2}=\left(\operatorname{AffineMap}\left(\frac{1}{a-(a-b)},-\frac{a-b}{a-(a-b)}\right)\right) \uparrow[a-b, a]$.

Set $f_{3}=\left(\operatorname{AffineMap}\left(-\frac{1}{a+b-a}, \frac{a+b}{a+b-a}\right)\right)\left\lceil[a, a+b]\right.$. Set $F=\left(f_{1}+\cdot f_{2}\right)+\cdot f_{3}$. $F(x)=\max \left(0,1-\left|\frac{x-a}{b}\right|\right)$.

Let us consider real numbers $a, b$ and a fuzzy set $f$ of $\mathbb{R}$. Now we state the propositions: 
(66) If $b>0$ and for every real number $x, f(x)=\max \left(0,1-\left|\frac{x-a}{b}\right|\right)$, then $f$ is triangular. The theorem is a consequence of (65).

(67) If $b>0$ and for every real number $x, f(x)=\max \left(0,1-\left|\frac{x-a}{b}\right|\right)$, then $f$ is strictly normalized.

Proof: There exists an element $x$ of $\mathbb{R}$ such that $f(x)=1$ and for every element $y$ of $\mathbb{R}$ such that $f(y)=1$ holds $y=x$.

(68) Let us consider a function $f$ from $\mathbb{R}$ into $\mathbb{R}$, and real numbers $a, b, c$. Suppose for every real number $x, f(x)=\max \left(0, \min \left(1, c \cdot\left(1-\left|\frac{x-a}{b}\right|\right)\right)\right)$. Then $f$ is a fuzzy set of $\mathbb{R}$. The theorem is a consequence of (25).

(69) Let us consider a function $f$ from $\mathbb{R}$ into $\mathbb{R}$, and real numbers $a, b$. Suppose $b>0$ and for every real number $x, f(x)=\max \left(0,1-\left|\frac{x-a}{b}\right|\right)$. Then $f$ is continuous.

PRoOF: $f=$ TriangularFS $((a-b), a,(a+b))$.

(70) Let us consider a function $f$ from $\mathbb{R}$ into $\mathbb{R}$, and real numbers $a, b, c$, $r, s$. Suppose $b \neq 0$ and for every real number $x, f(x)=\max (r, \min (s, c$. $\left.\left.\left(1-\left|\frac{x-a}{b}\right|\right)\right)\right)$. Then $f$ is Lipschitzian.

PROOF: There exists a real number $r$ such that $0<r$ and for every real numbers $x_{1}, x_{2}$ such that $x_{1}, x_{2} \in \operatorname{dom} f$ holds $\left|f\left(x_{1}\right)-f\left(x_{2}\right)\right| \leqslant r \cdot\left|x_{1}-x_{2}\right|$.

(71) Let us consider a function $f$ from $\mathbb{R}$ into $\mathbb{R}$, and real numbers $a, b, c$. Suppose $b \neq 0$ and for every real number $x, f(x)=\max (0, \min (1, c \cdot(1-$ $\left.\left.\left.\left|\frac{x-a}{b}\right|\right)\right)\right)$. Then $f$ is Lipschitzian.

(72) $\{f$, where $f$ is a function from $\mathbb{R}$ into $\mathbb{R}, a, b$ are real numbers : $b>$ 0 and for every real number $\left.x, f(x)=\max \left(0,1-\left|\frac{x-a}{b}\right|\right)\right\} \subseteq$ Membership$\operatorname{Funcs}(\mathbb{R})$.

Proof: $\{f$, where $f$ is a function from $\mathbb{R}$ into $\mathbb{R}, a, b$ are real numbers : $b>0$ and for every real number $\left.x, f(x)=\max \left(0,1-\left|\frac{x-a}{b}\right|\right)\right\} \subseteq$ $\{$ TriangularFS $(a, b, c)$, where $a, b, c$ are real numbers : $a<b<c\}$.

(73) $\{f$, where $f$ is a function from $\mathbb{R}$ into $\mathbb{R}, a, b, c, d$ are real numbers $: b \neq$ 0 and for every real number $\left.x, f(x)=\max \left(0, \min \left(1, c \cdot\left(1-\left|\frac{x-a}{b}\right|\right)\right)\right)\right\} \subseteq$ Membership-Funcs $(\mathbb{R})$. The theorem is a consequence of $(68)$.

(74) Let us consider real numbers $a, b, p, q, s$.

Then $(\operatorname{AffineMap}(a, b)) \uparrow]-\infty, s[+\cdot(\operatorname{AffineMap}(p, q)) \uparrow[s,+\infty[$ is a function from $\mathbb{R}$ into $\mathbb{R}$.

(75) Let us consider real numbers $a, b, p, q$, and a function $f$ from $\mathbb{R}$ into $\mathbb{R}$. Suppose for every real number $x, f(x)=$ $\max \left(0, \min (1,((\operatorname{AffineMap}(a, b)))]-\infty, \frac{q-b}{a-p}\left[+\cdot(\operatorname{AffineMap}(p, q)) \uparrow\left[\frac{q-b}{a-p}\right.\right.\right.$, $+\infty[(x)))$. Then $f$ is a fuzzy set of $\mathbb{R}$. The theorem is a consequence of (74) and (25). 
(76) Let us consider real numbers $a, b, c$. Suppose $a<b<c$. Then

(i) (TriangularFS $(a, b, c))(a)=0$, and

(ii) (TriangularFS $(a, b, c))(b)=1$, and

(iii) (TriangularFS $(a, b, c))(c)=0$.

(77) Let us consider real numbers $a, b, c, d$. Suppose $a<b<c<d$. Then

(i) (TrapezoidalFS $(a, b, c, d))(a)=0$, and

(ii) (TrapezoidalFS $(a, b, c, d))(b)=1$, and

(iii) (TrapezoidalFS $(a, b, c, d))(c)=1$, and

(iv) (TrapezoidalFS $(a, b, c, d))(d)=0$.

Let us consider real numbers $a, b, p, q$ and a real number $x$. Now we state the propositions:

(78) Suppose $a>0$ and $p>0$ and $\frac{-b}{a}<\frac{q}{p}$ and $\frac{1-b}{a}=\frac{1-q}{-p}$. Then (TriangularFS $\left.\left(\frac{-b}{a}, \frac{1-b}{a}, \frac{q}{p}\right)\right)(x)=\max \left(0, \min \left(1,((\operatorname{AffineMap}(a, b)) \Gamma]-\infty, \frac{q-b}{a+p}[+\cdot(\right.\right.$ Affine$\operatorname{Map}(-p, q))\left\lceil\left[\frac{q-b}{a+p},+\infty[)(x)\right)\right)$.

Proof: For every real number $x$, (TriangularFS $\left.\left(\frac{-b}{a}, \frac{1-b}{a}, \frac{q}{p}\right)\right)(x)=$ $\max \left(0, \min \left(1,((\operatorname{AffineMap}(a, b)) \uparrow]-\infty, \frac{q-b}{a+p}\left[+\cdot(\operatorname{AffineMap}(-p, q))\left\lceil\left[\frac{q-b}{a+p}\right.\right.\right.\right.\right.$, $+\infty[(x)))$.

(79) Suppose $a>0$ and $p>0$ and $\frac{1-b}{a}<\frac{1-q}{-p}$. Then (TrapezoidalFS $\left.\left(\frac{-b}{a}, \frac{1-b}{a}, \frac{1-q}{-p}, \frac{q}{p}\right)\right)(x)=$ $\max \left(0, \min \left(1,\left((\operatorname{AffineMap}(a, b))\lceil]-\infty, \frac{q-b}{a+p}\left[+\cdot(\operatorname{AffineMap}(-p, q))\left\lceil\left[\frac{q-b}{a+p}\right.\right.\right.\right.\right.\right.$, $+\infty[)(x)))$.

Proof: Set $\left.f_{4}=(\operatorname{AffineMap}(a, b)) \uparrow\right]-\infty, \frac{q-b}{a+p}[$.

Set $f_{5}=(\operatorname{AffineMap}(-p, q)) \uparrow\left[\frac{q-b}{a+p},+\infty[\right.$.

For every real number $x$, (TrapezoidalFS $\left.\left(\frac{-b}{a}, \frac{1-b}{a}, \frac{1-q}{-p}, \frac{q}{p}\right)\right)(x)=$ $\max \left(0, \min \left(1,\left(f_{4}+\cdot f_{5}\right)(x)\right)\right)$.

(80) Let us consider real numbers $a, b, p, q$, and a function $f$ from $\mathbb{R}$ into $\mathbb{R}$. Suppose $a>0$ and $p>0$ and $f=(\operatorname{AffineMap}(a, b)) \uparrow]-\infty, \frac{q-b}{a+p}[+\cdot($ Affine$\operatorname{Map}(-p, q))\left\lceil\left[\frac{q-b}{a+p},+\infty[\right.\right.$. Then $f$ is Lipschitzian.

Proof: There exists a real number $r$ such that $0<r$ and for every real numbers $x_{1}, x_{2}$ such that $x_{1}, x_{2} \in \operatorname{dom} f$ holds $\left|f\left(x_{1}\right)-f\left(x_{2}\right)\right| \leqslant r \cdot\left|x_{1}-x_{2}\right|$.

(81) Let us consider real numbers $a, b, p, q$. Suppose $a>0$ and $p>0$. Then there exists a real number $r$ such that

(i) $0<r$, and 
(ii) for every real numbers $x_{1}, x_{2}$ such that $x_{1}, x_{2} \in$ $\operatorname{dom}((\operatorname{AffineMap}(a, b))\rceil]-\infty, \frac{q-b}{a+p}\left[+\cdot(\operatorname{AffineMap}(-p, q)) \uparrow\left[\frac{q-b}{a+p},+\infty[)\right.\right.$ holds $\mid((\operatorname{AffineMap}(a, b)) \uparrow]-\infty, \frac{q-b}{a+p}\left[+\cdot(\operatorname{AffineMap}(-p, q)) \uparrow\left[\frac{q-b}{a+p},+\infty[)\right.\right.$ $\left(x_{1}\right)-((\operatorname{AffineMap}(a, b)) \uparrow]-\infty, \frac{q-b}{a+p}\left[+\cdot(\operatorname{AffineMap}(-p, q)) \uparrow\left[\frac{q-b}{a+p},+\infty[)\right.\right.$ $\left(x_{2}\right)|\leqslant r \cdot| x_{1}-x_{2} \mid$.

The theorem is a consequence of (74) and (80).

(82) Let us consider real numbers $a, b, p, q, r, s$, and a function $f$ from $\mathbb{R}$ into $\mathbb{R}$. Suppose $a>0$ and $p>0$ and for every real number $x, f(x)=$ $\max \left(r, \min \left(s,((\operatorname{AffineMap}(a, b)) \uparrow]-\infty, \frac{q-b}{a+p}\left[+\cdot(\operatorname{AffineMap}(-p, q)) \uparrow\left[\frac{q-b}{a+p}\right.\right.\right.\right.$, $+\infty[(x)))$. Then $f$ is Lipschitzian. The theorem is a consequence of $(74)$, $(81)$, and (1).

(83) Let us consider real numbers $a, b, c$. Suppose $a<b<c$. Let us consider a real number $x$. Then (TriangularFS $(a, b, c))(x)=$ $\max \left(0, \min \left(1,\left(\left(\operatorname{AffineMap}\left(\frac{1}{b-a},-\frac{a}{b-a}\right)\right)\lceil]-\infty\right.\right.\right.$, $b\left[+\cdot\left(\operatorname{AffineMap}\left(-\frac{1}{c-b}, \frac{c}{c-b}\right)\right) \uparrow[b,+\infty[)(x))\right)$. The theorem is a consequence of $(78)$.

(84) Let us consider real numbers $a, b, c$, $d$. Suppose $a<b<c<d$. Let us consider a real number $x$. Then (TrapezoidalFS $(a, b, c, d))(x)=$ $\max \left(0, \min \left(1,\left(\left(\operatorname{AffineMap}\left(\frac{1}{b-a},-\frac{a}{b-a}\right)\right) \uparrow\right]-\infty, \frac{b \cdot d-a \cdot c}{d-c+b-a}[+\cdot(\right.\right.$ AffineMap $\left.\left(-\frac{1}{d-c}, \frac{d}{d-c}\right)\right)\left\lceil\left[\frac{b \cdot d-a \cdot c}{d-c+b-a},+\infty[)(x)\right)\right)$. The theorem is a consequence of (79).

(85) Let us consider real numbers $a, b, p, q$, and a function $f$ from $\mathbb{R}$ into $\mathbb{R}$. Suppose $a>0$ and $p>0$ and for every real number $x, f(x)=$ $\max \left(0, \min \left(1,((\operatorname{AffineMap}(a, b)) \uparrow]-\infty, \frac{q-b}{a+p}\left[+\cdot(\operatorname{AffineMap}(-p, q)) \uparrow\left[\frac{q-b}{a+p}\right.\right.\right.\right.$, $+\infty[)(x)))$. Then $f$ is Lipschitzian.

(86) Let us consider real numbers $a, b, c$. If $a<b<c$, then TriangularFS $(a, b, c)$ is Lipschitzian. The theorem is a consequence of (83) and (82).

(87) Let us consider real numbers $a, b, c, d$. If $a<b<c<d$, then Trapezoidal$\mathrm{FS}(a, b, c, d)$ is Lipschitzian. The theorem is a consequence of (84) and (82).

Let us consider real numbers $a, b, p, q$ and a fuzzy set $f$ of $\mathbb{R}$. Now we state the propositions:

(88) Suppose $a>0$ and $p>0$ and $\frac{-b}{a}<\frac{q}{p}$ and $\frac{1-b}{a}=\frac{1-q}{-p}$ and for every real number $x, f(x)=\max \left(0, \min (1,((\operatorname{AffineMap}(a, b)))]-\infty, \frac{q-b}{a+p}[+\cdot(\right.$ Affine$\left.\operatorname{Map}(-p, q)) \uparrow\left[\frac{q-b}{a+p},+\infty[)(x)\right)\right)$. Then $f$ is triangular and strictly normalized. The theorem is a consequence of (78).

(89) Suppose $a>0$ and $p>0$ and $\frac{1-b}{a}<\frac{1-q}{-p}$ and for every real number $x, f(x)=\max \left(0, \min (1,((\operatorname{AffineMap}(a, b)))]-\infty, \frac{q-b}{a+p}[+\cdot\right.$ (AffineMap 
$(-p, q)) \uparrow\left[\frac{q-b}{a+p},+\infty[(x))\right)$. Then $f$ is trapezoidal and normalized. The theorem is a consequence of $(79)$.

(90) $\{f$, where $f$ is a fuzzy set of $\mathbb{R}: f$ is triangular $\} \subseteq \operatorname{Membership-Funcs}(\mathbb{R})$.

(91) $\{$ TriangularFS $(a, b, c)$, where $a, b, c$ are real numbers : $a<b<c\} \subseteq$ $\operatorname{Membership-Funcs}(\mathbb{R})$.

(92) $\{f$, where $f$ is a fuzzy set of $\mathbb{R}: f$ is trapezoidal $\} \subseteq$ Membership-Funcs $(\mathbb{R})$.

(93) \{TrapezoidalFS $(a, b, c, d)$, where $a, b, c, d$ are real numbers : $a<b<c<$ $d\} \subseteq$ Membership-Funcs $(\mathbb{R})$.

\section{REFERENCES}

[1] Grzegorz Bancerek, Czesław Byliński, Adam Grabowski, Artur Korniłowicz, Roman Matuszewski, Adam Naumowicz, Karol Pąk, and Josef Urban. Mizar: State-of-the-art and beyond. In Manfred Kerber, Jacques Carette, Cezary Kaliszyk, Florian Rabe, and Volker Sorge, editors, Intelligent Computer Mathematics, volume 9150 of Lecture Notes in Computer Science, pages 261-279. Springer International Publishing, 2015. ISBN 978-3319-20614-1. doi:10.1007/978-3-319-20615-8_17.

[2] Grzegorz Bancerek, Czesław Byliński, Adam Grabowski, Artur Korniłowicz, Roman Matuszewski, Adam Naumowicz, and Karol Pąk. The role of the Mizar Mathematical Library for interactive proof development in Mizar. Journal of Automated Reasoning, 61(1):9-32, 2018. do1:10.1007/s10817-017-9440-6

[3] Adam Grabowski. The formal construction of fuzzy numbers. Formalized Mathematics, 22(4):321-327, 2014. doi $10.2478 /$ forma-2014-0032.

[4] Adam Grabowski and Takashi Mitsuishi. Initial comparison of formal approaches to fuzzy and rough sets. In Leszek Rutkowski, Marcin Korytkowski, Rafal Scherer, Ryszard Tadeusiewicz, Lotfi A. Zadeh, and Jacek M. Zurada, editors, Artificial Intelligence and Soft Computing - 14th International Conference, ICAISC 2015, Zakopane, Poland, June 14-18, 2015, Proceedings, Part I, volume 9119 of Lecture Notes in Computer Science, pages 160-171. Springer, 2015. doi:10.1007/978-3-319-19324-3_15

[5] Artur Korniłowicz and Yasunari Shidama. Inverse trigonometric functions arcsin and arccos. Formalized Mathematics, 13(1):73-79, 2005.

[6] Bo Li, Yanhong Men, Dailu Li, and Xiquan Liang. Basic properties of periodic functions. Formalized Mathematics, 17(4):245-248, 2009. doi 10.2478/v10037-009-0031-9.

[7] E. H. Mamdani. Application of fuzzy algorithms for control of simple dynamic plant IEE Proceedings, 121:1585-1588, 1974.

[8] Takashi Mitsuishi. Uncertain defuzzified value of periodic membership function. In 2018 International Electrical Engineering Congress (iEECON), pages 1-4, 2018. doi: 10.1109 /IEECON.2018.8712319

[9] Takashi Mitsuishi, Noboru Endou, and Yasunari Shidama. The concept of fuzzy set and membership function and basic properties of fuzzy set operation Formalızed Mathematıcs, $9(2): 351-356,2001$.

[10] Takashi Mitsuishi, Katsumi Wasaki, and Yasunari Shidama. Basic properties of fuzzy set operation and membership function. Formalized Mathematics, y(2):357-362, 2001.

[11] Takashi Mitsuishi, Noboru Endou, and Keiji Ohkubo. Trigonometric functions on complex space. Formalized Mathematics, 11(1):29-32, 2003.

[12] Takashi Mitsuishi, Takanori Terashima, Nami Shimada, Toshimichi Homma, Kiyoshi Sawada, and Yasunari Shidama. Continuity of defuzzification on $\mathrm{L}^{2}$ space for optimization of fuzzy control. In Active Media Technology, pages 73-81. Springer-Berlin-Heidelberg, 2012. ISBN 978-3-642-35236-2.

[13] Takashi Mitsuishi, Nami Shimada, Toshimichi Homma, Mayumi Ueda, Masayuki Kochizawa, and Yasunari Shidama. Continuity of approximate reasoning using fuzzy number under Łukasiewicz t-norm. In 2015 IEEE 7th International Conference on Cybernetics 
and Intelligent Systems (CIS) and IEEE Conference on Robotics, Automation and Mechatronics (RAM), pages 71-74, 2015. doi $10.1109 /$ ICCIS.2015.7274550.

Accepted June 30, 2021 